\title{
EL DENGUE EN EL NORORIENTE DE VENEZUELA Y SU INCIDENCIA EN CONDICIONES EXTREMAS DE LAS LLUVIAS DURANTE LOS AÑOS 2009 Y $2010^{1}$
}

\author{
Vidal Sáez Sáez y Luis A. Colmenares Suárez ${ }^{3}$ \\ Postgrado de la Facultad de Humanidades y Educación ${ }^{2}$ \\ Cátedra de Medicina Tropical, Facultad de Medicina ${ }^{3}$ \\ Universidad Central de Venezuela
}

\section{RESUMEN}

El objetivo fue evaluar comparativamente la incidencia de dengue, la lluvia de los años 2009 y 2010 y el servicio de abastecimiento de agua potable a la población de la región nororiental del país. Considerando los registros de casos de dengue de los años 2009 y 2010, se llevaron a incidencia (casos/total de población*10.000 habitantes) y se compararon con registros históricos para la región y el país. Al contrastar las lluvias de los años 2009 y 2010 con los históricos de la región (en proporción) y el país determinamos las diferencias observadas. Por otra parte, y con el objeto de vincular el comportamiento de las lluvias de estos años con el índice de oscilación del sur (IOS; bloque del Pacifico 3,4) se identifican por analogía relaciones entre las variables. Examinamos el abastecimiento de los servicios de agua potable y frecuencia, en centros poblados rurales y urbanos, como un indicador que determina en la presencia del vector. Elaboramos una síntesis de las relaciones establecidas en una realidad geográfica oriental con el comportamiento de las lluvias de los dos años que permiten caracterizar la persistencia de la enfermedad. Conclusión: las reservas de agua manipuladas por el hombre, a causa de la irregularidad del servicio de suministro a la población, y la afectación por eventos externos han sido algunos de los factores determinantes en la incidencia del dengue y se debe ampliar la evaluación de un conjunto de variables vinculadas a este hecho geográfico.

Palabras clave: geografía, dengue, lluvia, agua, población, Venezuela

\section{ABSTRACT}

\section{Dengue in the North Easter region of Venezuela and its incidence in extreme conditions of rains during 2009 and 2010}

The objective was to evaluate the incidence of dengue, the rain of the years 2009 and 2010 and the water supply service to the population of this North Easter region. Records in the years 2009 and 2010 were considered in the cases of dengue were carried incidence (cases / total population of 10,000) and compared with historical records for both the North Easter region and the country, comparing the rains during the years 2009 and 2010, with the historic of the region and the country to determine the observed differences. On the other hand, and in order to link the behavior of these years rains characterized the Southern Oscillation Index (SOI: block Pacific 3.4) with the object identified by analogy relationships between variables. Revised supply of drinking water in the region and often in rural towns and urban

\footnotetext{
Este trabajo fue posible gracias al financiamiento del Consejo de Desarrollo Humanístico y Científico (CDCH) de la Universidad Central de Venezuela (UCV), bajo el número PI-07-8364-2011/1 y la Sociedad Venezolana de Infectología.

2 Contacto: vidal.saezsaez@gmail.com

Contacto: lacsuarez@yahoo.com
} 
centers, as an indicator in determining the presence of the vector. Finally, a synthesis of the relationships established in a geographical North Easter and rainfall patterns of those two years that characterized the persistence of the disease. We conclude that the water reserves manipulated by man, because of the irregularity of supply, as a service to the public and also affected by external events has been one of the determining factors in the incidence of dengue and broadening the assessment of a group of variables related to this geographical fact.

Keywords: geography, dengue, rain, water, population, Venezuela

\section{INTRODUCCIÓN}

La ocurrencia de dengue obedece a diversos factores: entre ellos, la misma complejidad en la que el virus hace sus recorridos en diversos medios y hospedadores, la presencia de un vector que lo transmite, las condiciones del medio (temperatura del aire y humedad) que permiten la existencia del virus, y, en particular, la supervivencia del vector; también hay que agregar el factor humano, ya que el manejo y almacenamiento de agua potable, así como las condiciones de los servicios de salubridad para la población inciden en la presencia del vector que porta la enfermedad. La región nororiental de Venezuela, integrada por los estados Anzoátegui, Delta Amacuro, Monagas, Nueva Esparta y Sucre, está emplazada al este del país y tiene una superficie de $125.350 \mathrm{~km}^{2}$ en paisajes que van desde llanuras, colinas, piedemonte y montañas, con elevaciones entre 0 a más de 1800 metros sobre nivel mar (Ministerio del Ambiente y de los Recursos Naturales Renovables: MARNR; 1983). Según el Instituto Nacional de Estadística, INE, (2012) cuenta con una población de 3.930.767 habitantes.

Los años 2009 y 2010 se presentan como extremos en el comportamiento de las lluvias en país, producto de la acción de diversas condiciones del sistema climático regional, entre ellos la alternancia de un evento de El Niño y La Niña en el Pacífico, junto a otras variables macroclimáticas tropicales. También en esos dos años ocurrieron los más altos registros de casos de dengue en Venezuela (Ministerio Popular para la Salud: MPPS, 2012), y en cierta medida en la región nororiental. La temporada seca del año 2008 en promedio se inició en octubre/ noviembre y se extendió de manera irregular durante todo el año 2009. Los valores observados estuvieron por debajo de lo normal, esto afectó las múltiples actividades de la sociedad (como las agropecuarias, la producción hidroeléctrica), las reservas y suministro de agua.

Las lluvias del año 2010 se inician en término promedio a la fecha, luego de varias semanas los montos y frecuencias de las precipitaciones se presentan muy por encima a los valores promedios durante todo ese período, esta situación provoca inundaciones, deslaves, alteración de las actividades humanas y afectación de los servicios básicos a la población (El Nacional, 2011; El Universal, 2011; González, 2010) siendo, a su vez, los años con mayor número de casos de dengue observados en décadas (MPPS, 2012). Se destaca entonces en las situaciones descritas tres elementos en común: los altos valores de incidencia de dengue en el período, un comportamiento extremo de las lluvias y la afectación de los servicios a la población, a causa de déficit o exceso de humedad de las precipitaciones registradas.

\section{OBJETIVO}

Evaluar comparativamente la incidencia de dengue, la lluvia de los años 2009 y 2010 y el servicio de abastecimiento de agua potable a la población de la región nororiental de Venezuela.

\section{METODOLOGÍA}

Debido la complejidad de las variables que deben ser tomadas en cuenta para la ocurrencia del dengue, y lograr comprenderla desde el enfoque geográfico, a partir de un análisis espacial, se debe considerar cada uno de los componentes que la identifican en una evaluación individual, para lograr al final del estudio una síntesis integradora que permita concluir en el reconocimiento de la enfermedad como un hecho geográfico. 
a) En primer lugar, los datos registrados de casos de dengue suministrados por el Ministerio del Poder Popular Para la Salud (MPPS, 2012) y anterior Ministerio de Salud y Desarrollo Social (MSDS; 2006) para los años 2009 y 2010, se llevaron a índices o en incidencia (casos/total de población*10.000 habitantes); luego, se compararon las incidencias con registros históricos para la región oriental y para el país, con el objeto de evaluar la magnitud de la afectación.

b) En segundo lugar, y como otro de los componentes que permiten la persistencia de la enfermedad, se estudió el comportamiento de las precipitaciones. A partir de las diferencias proporcionales de la información suministrada por el Instituto Nacional de Meteorología e Hidrología, INAMEH, (2012) y Hernández (2011), se comparan los registros de lluvias de los años 2009 y 2010 con los registros históricos de la región y el país, de manera de establecer las diferencias. Por otra parte, y con el objeto de vincular el comportamiento de las lluvias de estos años con variables extrarregionales, se caracteriza el índice de oscilación del sur (IOS) correspondiente al bloque del Pacífico 3,4. El IOS es uno de los indicadores que mide el ENOS (El Niño-Oscilación del Sur). Es una variable atmosférica de control que junto al indicador (registros mensuales) de la Anomalía de la Temperatura Superficial del Mar (SSTA, en inglés) en el Océano Pacífico ecuatorial, los registros están disponibles en la NOAA (2012).

c) Como tercer componente de apreciable vinculación es la forma de identificar los reservorios de agua dulce, sea por lluvias o por almacenamiento, dado que el mosquito requiere estos cuerpos de agua para asegurar su supervivencia; es así que se hace una revisión de la presencia, y tipo de los servicios de abastecimiento agua potable, en centros poblados, rurales y urbanos, de los estados que conforman la región oriental del país y se hace referencia a la frecuencia del servicio (INE, 2012).

d) Finalmente, se hace una síntesis de las relaciones que se establecen en una realidad geográfica oriental y del comportamiento de las lluvias de los dos años que permite caracterizar la persistencia de la enfermedad.

\section{RESULTADOS}

A continuación se hace una presentación del comportamiento del dengue en la región oriental en Venezuela, durante los años 2009 y 2010, y las variables asociadas a su ocurrencia.

La región se emplaza al este del país. Limita al norte con el Mar Caribe; al sur con el estado Bolívar; por el este con Trinidad y el océano Atlántico, y al oeste con los estados Miranda y Guárico. Tiene elevaciones de 0 a 2.610 metros sobre el nivel del mar (figura 1). Esta región presenta espacios geográficos bien diferenciados. Al norte, el relieve está dominado por formaciones montañosas, se caracteriza por presentar la vertiente norte muy abrupta, mientras que el sur es más suave. Al noreste en su mayoría es plano, tiene una llanura costera sobre el Mar Caribe y al sureste, la llanura fluvial sobre el río Orinoco (MARNR, 1983). La población de la región nororiental representa el 15 \% del total nacional (INE, 2012).

\subsection{Incidencia del dengue}

Venezuela es un país tropical, y existe la incidencia de enfermedades vinculadas a este ámbito geográfico. Entre ellas, el dengue -una enfermedad que afecta al hombre y puede ser mortal en su forma severa (Organización Panamericana de la Salud, OPS, 2011 y 2008)-, ocasionada por un virus y transmitida por la picadura de un insecto o mosquito (Aedes aegypti). Cifras del Ministerio de Salud indican un comportamiento creciente de la incidencia de dengue en el país (MPPS, 2012; MSDS, 2006). Durante el periodo de 1965 hasta 1994, la incidencia de la enfermedad en Venezuela fue muy baja (MSDS, 2006), los valores observados, en término promedio, no pasaban de cinco infectados por cada 10000 habitantes. Luego, a partir de 1997 hasta la fecha se observa una tendencia oscilante en los casos ocurridos y hace que la incidencia se haya incrementado en los últimos seis años con valores para 2009 de 23,6 hab/10000hab y en 2010 de 44,7 en Venezuela (tabla 1). 
Figura 1. Localización relativa de la región Nororiente en Venezuela.

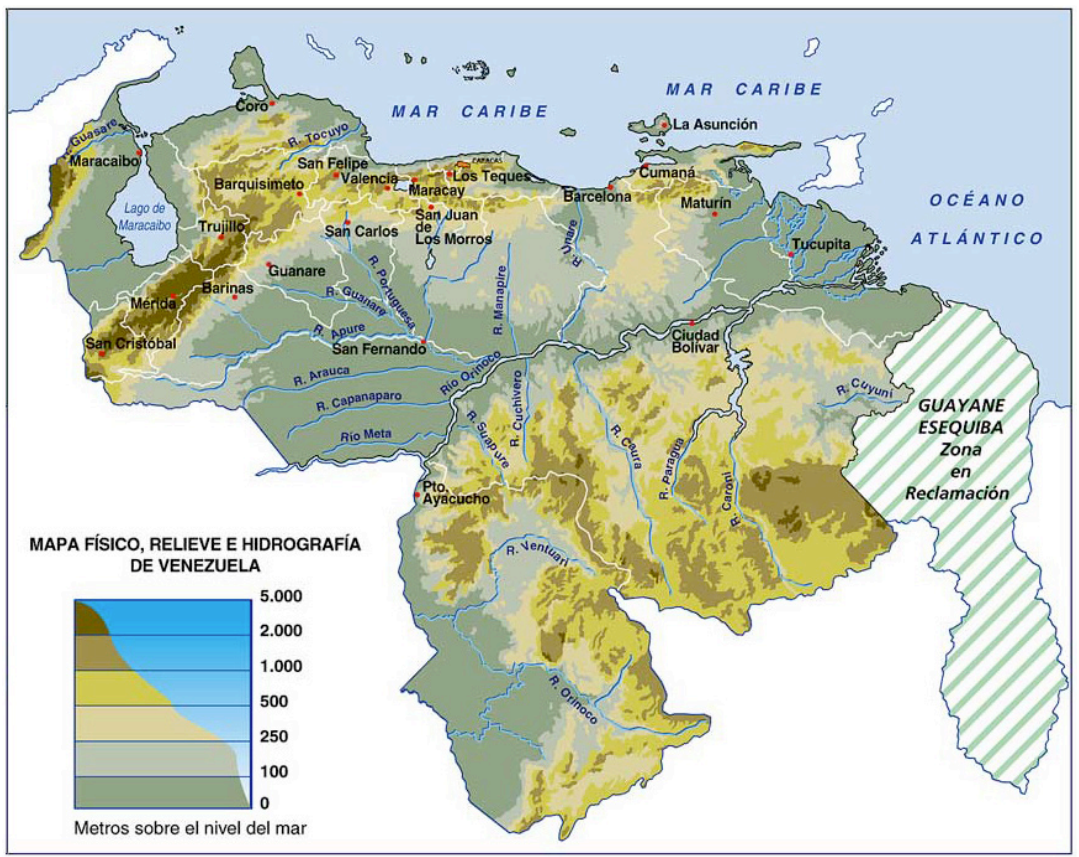

Fuente: http://www.a-venezuela.com/mapas/map/html/relieve.html

Por otra parte, la región nororiental es un espacio con diversidad de paisajes (MARNR, 1983), lo que hace que las condiciones del medio favorezcan la presencia del mosquito que transmite la enfermedad; los espacios con considerables alturas, provocan que la temperatura del aire baje y cuando los valores son muy templados inciden en la presencia del mosquito, no es el caso en esta situación.

Tabla 1. Incidencia (casos/pob. total*10000 habitantes) de dengue en Venezuela y la región Nororiental. Años 2000 a 2010.

\begin{tabular}{|c|c|c|c|c|c|c|c|c|c|c|c|}
\hline & 2000 & 2001 & 2002 & 2003 & 2004 & 2005 & 2006 & 2007 & 2008 & 2009 & 2010 \\
\hline Venezuela & 8,7 & 33,7 & 15,3 & 10,6 & 11,8 & 15,9 & 15,5 & 29,1 & 17,2 & 23,6 & 44,7 \\
\hline Oriente & 7,1 & 12,5 & 2,4 & 1,9 & 1,5 & 1,0 & 1,9 & 15,7 & 7,9 & 9,0 & 14,5 \\
\hline Anzoátegui & 13,8 & 24,4 & 4,7 & 3,8 & 3,0 & 1,9 & 3,7 & 31,0 & 15,6 & 17,8 & 28,8 \\
\hline Delta Amacuro & 0,7 & 17,8 & 4,7 & 7,1 & 4,4 & 7,6 & 26,0 & 29,1 & 1,7 & 14,1 & 31,5 \\
\hline Monagas & 10,2 & 53,4 & 15,1 & 15,8 & 16,1 & 11,8 & 11,3 & 40,8 & 24,8 & 47,3 & 46,3 \\
\hline Nueva Esparta & 7,1 & 12,5 & 2,4 & 1,9 & 1,5 & 1,0 & 1,9 & 15,7 & 7,9 & 9,0 & 14,5 \\
\hline
\end{tabular}

Fuente: INE (2012), MPPS (2012), MSDS (2006).

En la tabla 1 se aprecia el comportamiento de la incidencia en el tiempo para Venezuela y para la región Nororiental del país. Los valores de estimación sugieren, tal como se ha mencionado, una tendencia para las series de información al incremento de los casos de afectados para el país (Orihuela, 2008a 2008b; Red de Sociedades Científicas Médicas Venezolanas, 2011) y para el nororiente, y comparativamente se puede señalar que la tasa de crecimiento es mayor para Venezuela.

Históricamente, al contrastar la incidencia regional con la nacional se tiene que en el área los índices casi siempre están por debajo que en el resto del país, la incidencia del año 2009 fue de 9,0 personas enfermas por cada 10000 habitantes y luego un considerable incremento para el año 2010, con un índice de 14,5. Lo que representa un alto valor observado a pesar de las medidas que se venían tomando en el país, en otras áreas en estado más crítico, y luego del repunte de casos ocurridos en el 2008 en Venezuela $(17,2$ personas/10.000 hab) y que de manera comparativa se puede concluir parcialmente que hubo un incremento de la incidencia en la población oriental. 
En la tabla también se puede apreciar los valores de la incidencia para cada estado que conforma la región Nororiental. De allí se revela que Monagas es la entidad con mayor incidencia en la región; incluso, sus registros señalan que los indicadores están por encima a los registros nacionales en casi todo el período considerado. Es necesario mencionar también que el patrón de incremento de la incidencia de dengue en el país no es un fenómeno exclusivo de nuestro contexto, las fluctuaciones ocurridas en los últimos años también han sido observadas en otros países de América Tropical (PAHO, 2009) pero las mayores tasas de incidencia se pueden apreciar en Venezuela (MPPS, 2012; Oletta, 2009).

\subsection{Las precipitaciones en la región nororiental}

Dado que en la región Nororiental destacan amplias diferencias de alturas: hacia el norte, con la serranía del Turimiquire, así como tiene orientación hacia el océano Atlántico; al este con el estado Delta Amacuro, hace que sus paisajes sean muy diversos (MARNR, 1983). Las precipitaciones dominan en buena parte del área los Llanos, en la parte oeste, centro y sur de la región oriental, y junto con la acción del viento y el movimiento de la franja de convergencia intertropical permiten, entre otras condiciones, que se formen espacios de concentración de lluvias. El resultado final es que en término promedio, las lluvias anuales oscilen entre menos de 600 a $800 \mathrm{~mm}$ al año, en la parte extrema noroeste de la región, es decir hacia las costas de Anzoátegui y parte de Sucre, en cambio, hacia el oeste y centro de la región, y en dirección al este, las lluvias promedios anuales oscilan entre 800 a más de $2000 \mathrm{~mm}$. En el área de la Serranía las lluvias varían entre 1800 hasta un poco más de $2000 \mathrm{~mm}$ de la precipitación anual (INAMEH, 2012).

El mes más seco de la región es marzo, en promedio caen menos de $5 \mathrm{~mm}$ de lluvia en ese período en la parte oeste, es decir, en Anzoátegui y parte central de Monagas; luego, se observa un incremento en el centro-sur, norte y este con un gradiente de va de más de $20 \mathrm{~mm}$ hasta más de $100 \mathrm{~mm}$ en las partes altas del Turimiquire y extremo este del Delta Amacuro. Junio y julio son en promedio los meses más lluviosos para la región, y con el mismo patrón observado en marzo (mes más seco), se tiene que hacia el noroeste los registros son de un poco menos de 80 a $100 \mathrm{~mm}$, se incrementa en la parte central y al sur en un promedio de 140 a $160 \mathrm{~mm}$; finalmente, al norte y extremo este de la región, las lluvias medias mensuales de junio a julio se cuantifican entre 200 a más de $300 \mathrm{~mm}$.

Al comparar los registros medios mensuales con las lluvias ocurridas en los años 2009 y 2010, se identifican situaciones extremas en el comportamiento regular de los valores observados de las precipitaciones. Es necesario señalar que las lluvias en Venezuela están determinadas en gran medida por la Zona de Convergencia Intertropical y estas pueden ser alteradas por variables macroclimáticas, tal como lo confirman Cárdenas et al., (2003) y Martelo (2003).

En primer lugar, se determina que el año 2009 fue un año seco al comparar las lluvias observadas con los registros medios; el año 2010 fue muy húmedo. En general, esto se vincula con una variable macroclimática extrarregional que afectó de igual manera a otros países sudamericanos, y fue la acción de la ocurrencia de un evento de El Niño (2009) seguido de manera inmediata por una Niña (IPCC, 2012; en español: Grupo Intergubernamental de Expertos sobre el Cambio Climático). En la tabla 2, se aprecian las diferencias porcentuales mensuales de las lluvias observadas en los años 2009 y 2010 con respecto a sus valores normales. En la figura 2, se aprecia la espacialización de las lluvias en Venezuela y la región nororiental y sus diferencias con respecto a los valores medios para los meses de julio del año 2009 y octubre de 2010.

Tabla 2. Variación porcentual (\%) de las lluvias observadas, años 2009 y 2010. Región Nororiental. Venezuela.

\begin{tabular}{|c|c|c|c|c|c|c|c|c|c|c|c|c|}
\hline Año & ene & feb & mar & abr & may & jun & jul & ago & sep & oct & nov & dic \\
\hline 2009 & -- & $\begin{array}{l}-60 \mathrm{al} \\
+160\end{array}$ & $\begin{array}{c}0 \text { al } \\
-100\end{array}$ & $\begin{array}{c}-25 \text { al } \\
-100\end{array}$ & -100 & $\begin{array}{l}-25 \text { al } \\
-100\end{array}$ & $\begin{array}{c}-25 \mathrm{al} \\
-75\end{array}$ & $\begin{array}{c}-25 \text { al } \\
-75\end{array}$ & $\begin{array}{c}-25 \mathrm{al} \\
-75\end{array}$ & $\begin{array}{c}25 \mathrm{al} \\
-75\end{array}$ & $\begin{array}{c}-25 \mathrm{al} \\
-75\end{array}$ & $\begin{array}{l}0 \mathrm{al} \\
-75\end{array}$ \\
\hline 2010 & $\begin{array}{l}+25 \\
-75\end{array}$ & -100 & $\begin{array}{c}-50 \mathrm{al} \\
-100\end{array}$ & $\begin{array}{l}0 \mathrm{al} \\
+50\end{array}$ & $\begin{array}{l}75 \mathrm{al} \\
+100\end{array}$ & $\begin{array}{c}-25 \mathrm{al} \\
+25\end{array}$ & $\begin{array}{c}0 \mathrm{al} \\
+100\end{array}$ & $\begin{array}{c}-25 \mathrm{al} \\
+75\end{array}$ & $\begin{array}{l}50 \mathrm{al} \\
+100\end{array}$ & $\begin{array}{c}-25 \mathrm{al} \\
+50\end{array}$ & $\begin{array}{c}+50 \mathrm{al} \\
+200\end{array}$ & $\begin{array}{c}+100 \mathrm{al} \\
200\end{array}$ \\
\hline
\end{tabular}

Fuente: Hernández, 2011. 
Para retomar la situación descrita de las lluvias en estos dos años, se tiene que desde marzo hasta diciembre del año 2009, las diferencias porcentuales de la precipitación observadas con respecto a los valores históricos, según registros del INAMEH (2012) y estimaciones hechas por Hernández (2011), fueron del -25 al $-75 \%$. Al examinar la espacialización de estas diferencias se tiene que tuvieron un comportamiento similar durante casi todos los meses del año 2009. Las mayores diferencias porcentuales (del 75 al -100\%) se apreciaron al extremo noroeste, costas de Anzoátegui, de la región oriental; al este, en el Delta Amacuro, se observaron las menores diferencias porcentuales con valores del - $25 \%$ de las lluvias; incluso en octubre y diciembre de 2009, en estas áreas los valores caídos eran similares a lo normal. Esta situación de sequía fue muy irregular en los registros del país, y no se observaba desde hacía muchos años (INAMEH, 2012), a tal punto que las diferencias fueron de -50\% en casi todo el país, momento que, en términos normales, es uno de los meses más lluviosos en Venezuela.

Luego, al observar los registros de lluvias mensuales del año 2010 (proporciones), y comparándolos con las series históricas, se determinó que el primer trimestre fue seco, como suele ser la temporada seca de la región y del país, pero con diferencias muy amplias - de menos del 25 al -100\% -, ello representaba la continuidad de la sequía registrada en 2009. El registro del mes de abril de 2010, para la región, y según la comparación, fue similar a lo normal, pero desde mayo la cantidad de precipitaciones ocurridas señalaban un exceso, muy por encima de lo normal, de manera que desde este momento y hasta agosto los valores estaban entre el 50 y $100 \%$ por encima del promedio, y en el último trimestre, específicamente en los meses de noviembre y diciembre, en la región nororiental, al igual que en casi todo el país, las lluvias estaban en más del $200 \%$ de variación proporcional a lo histórico.

Estas variaciones no son frecuentes y las fuentes especializadas señalaban que estos valores no ocurrían en el país desde hacía décadas. Una de las razones que hicieron que el patrón de las lluvias variara de esta manera en Venezuela en esos dos años, fue la acción de variables macroclimáticas, en este caso el ENSO (El Niño-Oscilación del Sur). Este evento se caracteriza por el calentamiento y enfriamiento anómalos de la superficie del agua en el océano Pacifico, y cuando se presenta tiene sus consecuencias sobre el sistema climático en América del Sur y el resto de América y áreas costeras de Asia. En esta oportunidad se presentó un evento moderado a intenso de Niño en el año 2009 y otro similar pero como Niña, en el 2010 (IPCC, 2012; Cárdenas et al.; Martelo, 2003).

Figura 2. Distribución espacial de las diferencias proporcionales de las lluvias ocurridas en los meses de julio de 2009 y octubre de 2010, en la región Oriental y Venezuela (Fuente: Hernández, 2011).
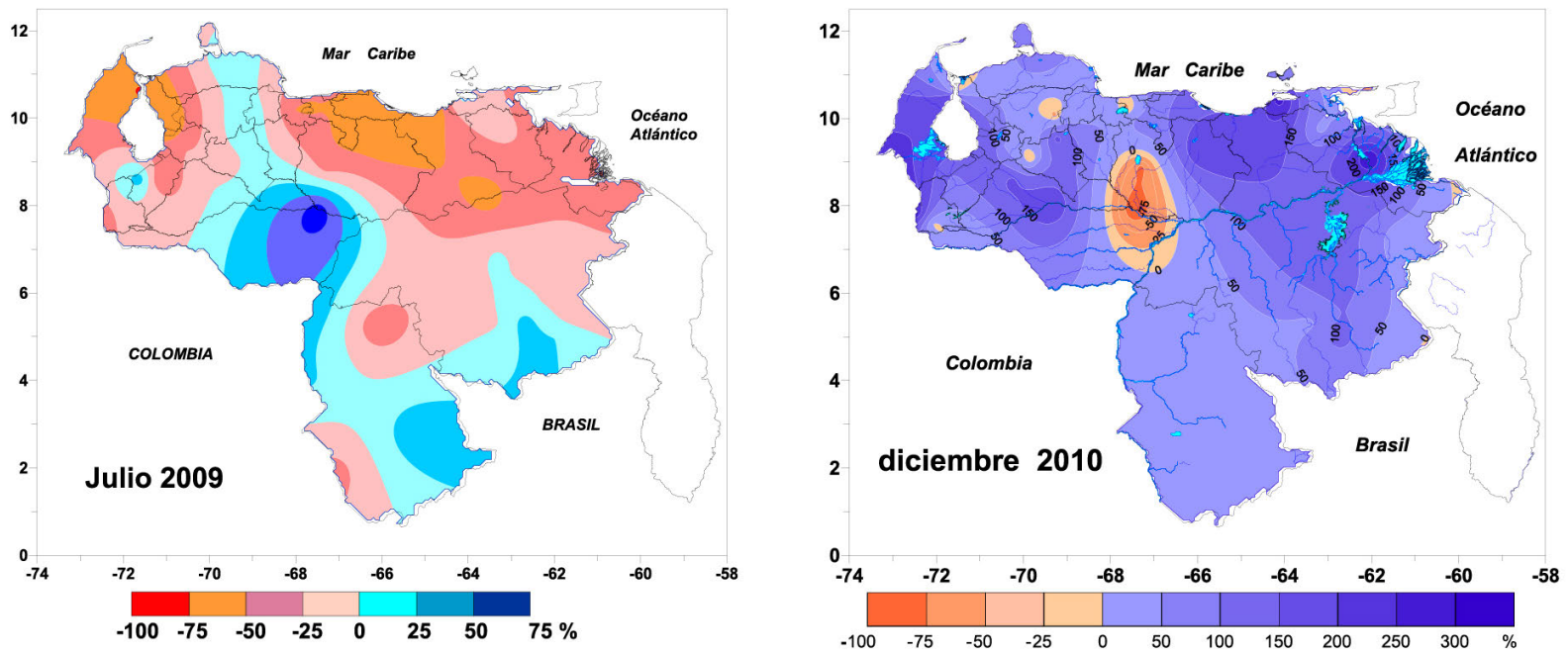

La medición de esta anomalía se puede considerar a través del registro de las desviaciones de los valores de la temperatura superficial del agua en el océano, lo cual lo convierte en un índice denominado Índice de Oscilación del Sur (IOS); las observaciones se realizan en varios bloques diferenciados en el 
océano. Para este trabajo se empleó el Ios perteneciente al espacio geográfico $3.4\left(5^{\circ} \mathrm{N}-5^{\circ} \mathrm{S} ; 170^{\circ}-120^{\circ} \mathrm{W}\right.$. en el Pacífico; en inglés se denomina SSTA 3.4). Por otra parte, estos valores tienen umbrales de intensidad y de allí la categorización que se indica en la tabla 3, a continuación (Cárdenas, 2003):

Tabla 3. Intensidad de ENOS (El Niño/Oscilación del Sur) en la región 3.4.

\begin{tabular}{|c|c|}
\hline Intensidad del evento & SSTA 3.4 \\
\hline Neutro & $>0,64$ \\
\hline Débil & $0.65-1.00$ \\
\hline Moderado & $1,00-1,50$ \\
\hline Fuerte & $>1,51$ \\
\hline
\end{tabular}

Fuente: Cárdenas (2003).

Los valores menores a 0,65 pueden llegar a ser negativos, fenómeno de condición contraria a El Niño, y se conoce como La Niña que afecta con la misma intensidad el sistema climático a nivel mundial (Organización Meteorológica Mundial: OMM, 2011; Cárdenas et al., 2003; Martelo 2003).

En la figura 3, se aprecian los valores mensuales de los desvíos de la temperatura superficial del agua en bloque pacifico 3,4 para los años 2008 al 2010. En 2009, se observa que hasta junio los registros eran neutros en sus desvíos, situación que ocurría desde mayo de 2008; luego de julio a octubre pasa a un evento débil, después el índice cambió de moderado a fuerte en un mes, y desde noviembre del año 2009 hasta febrero de 2010 se mantiene esa condición, es decir, un evento Niño de moderado a fuerte; a su vez, justo durante esos meses se presentó una sequía severa en casi todo el país, y afectó a la región nororiental de Venezuela.

Luego, en el año 2010 el océano Pacífico se enfría rápidamente entre marzo y mayo, y dio paso a un evento La Niña; el cual entre junio y julio, pasa de débil a moderado; en el mes de septiembre se transforma en un evento de intensidad fuerte que continua por el resto del año. En paralelo, las lluvias en nuestro país, en el último trimestre de ese año donde la temporada de precipitaciones ya ha pasado y se comienza a establecer el período seco, superaban de manera holgada los registros medios mensuales en dos y tres veces más en algunas regiones, mientras que en el nororiente de Venezuela las diferencias proporcionales estaban entre el 50 a más del $200 \%$.

Figura 3. Valores de desviación de la temperatura superficial del agua en el Pacífico (3,4). Años 2008-2010.

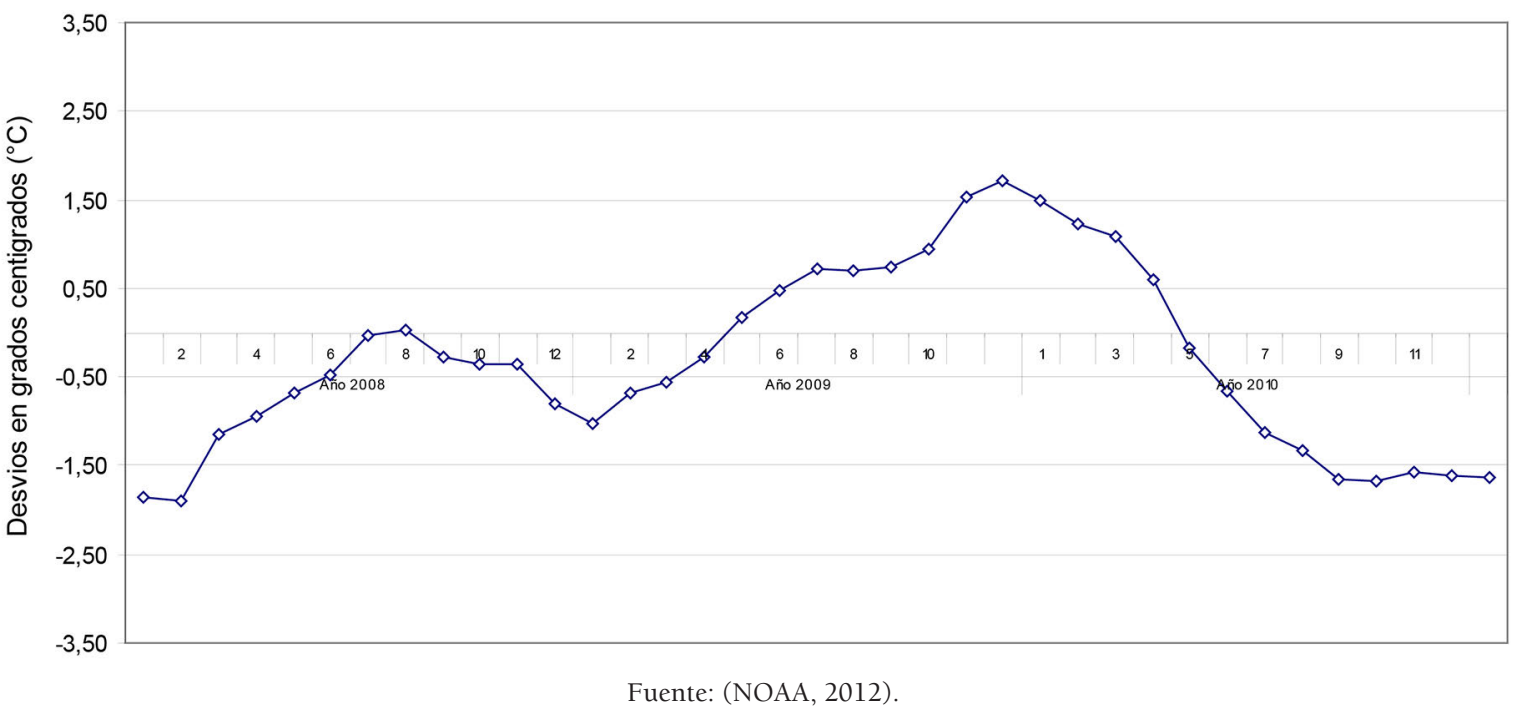


Por otra parte, se debe mencionar que la ocurrencia del ENSo durante los años 2009 y 2010 tuvo una acción directa y más severa en lluvias y sequías en América del Sur, en particular, con las precipitaciones ocurridas en Colombia y Bolivia, las sequías en Brasil e invierno severo en Chile (El Universal, 2011), situaciones meteorológicas que en Venezuela se tradujeron en desastres, afectando la dinámica del desempeño de las comunidades debido a la afectación de servicios a la población, daños en áreas de producción agrícola, inundaciones y deslaves, pérdidas de vidas y, por supuesto, registros extremos en incidencias de enfermedades asociadas a la situación de desastres.

\subsection{Servicio básico: abastecimiento de agua potable en la región Nororiental de Venezuela}

El dengue es una enfermedad que se vincula a la actividad del hombre, porque el abastecimiento de agua para el consumo humano se transforma en un medio favorable para el desarrollo de la larva del mosquito (OPS 2011; 2008). Las posibilidades de incremento de casos de infectados aumentan en la medida en que se improvisan nuevas formas de almacenar agua para el consumo, lo que facilita la reproducción de mosquitos portadores de la enfermedad. Por tanto, al aumentar las reservas de almacenamiento de agua, las cuales complementan el abastecimiento directo por tuberías o atenúan la frecuencia irregular del servicio es una variable de importancia, ya que puede convertirse en un medio favorable para la prevalencia e incremento de la población anofelina.

A partir de este supuesto, y en vista de las modalidades existentes de abastecimiento de servicios de agua potable, en la tabla 4, se mencionan los centros poblados y las entidades en las que se emplazan en la región. Existen, en el nororiente del país 2875 centros poblados con 643481 viviendas, según el INE (2012); que varían desde pequeños centros rurales hasta grandes aglomeraciones en las ciudades capitales. Se observa en la tabla, los tipos de abastecimiento de agua a la población. Según los registros, en promedio el $77,23 \%$ de las viviendas en los centros poblados descritos poseen abastecimiento de agua por acueducto o tubería.

Tabla 4. Servicio de agua potable, por entidad. Región de Oriente. Venezuela.

\begin{tabular}{|c|c|c|c|c|c|c|}
\hline & $\begin{array}{c}\text { Número } \\
\text { de centros } \\
\text { poblados }\end{array}$ & $\begin{array}{c}\text { Total } \\
\text { viviendas }\end{array}$ & $\begin{array}{c}\text { Acueducto o } \\
\text { tubería (\%) }\end{array}$ & $\begin{array}{c}\text { Camión } \\
\text { cisterna (\%) }\end{array}$ & $\begin{array}{c}\text { Pila pública } \\
\text { o estanque } \\
(\%)\end{array}$ & $\begin{array}{c}\text { Pozo con } \\
\text { tubería o } \\
\text { bomba (\%) }\end{array}$ \\
\hline Anzoátegui & 1328 & 376898 & 86,2 & 7,8 & 0,5 & 2,7 \\
\hline Delta Amacuro & 571 & 36987 & 55,9 & 10,7 & 0,6 & 3,3 \\
\hline Monagas & 898 & 217194 & 74,0 & 2,3 & 0,7 & 19,1 \\
\hline Nueva Esparta & 78 & 124023 & 92,8 & 3,8 & 1,0 & 0,5 \\
\hline
\end{tabular}

\begin{tabular}{|c|c|c|c|c|c|}
\hline & $\begin{array}{c}\text { Pozo o } \\
\text { manantial } \\
\text { protegido } \\
(\%)\end{array}$ & $\begin{array}{c}\text { Aljibes o } \\
\text { Jagüeyes } \\
(\%)\end{array}$ & $\begin{array}{c}\text { Río,caño, } \\
\text { quebrada } \\
(\%)\end{array}$ & $\begin{array}{c}\text { Lago, } \\
\text { laguna (\%) }\end{array}$ & $\begin{array}{c}\text { Otros } \\
\text { medios (\%) }\end{array}$ \\
\hline Anzoátegui & 0,2 & 0,2 & 0,8 & 0,4 & 1,1 \\
\hline Delta Amacuro & 0,3 & 4,2 & 19,4 & 0,5 & 5,1 \\
\hline Monagas & 0,8 & 0,6 & 0,7 & 0,0 & 1,7 \\
\hline Nueva Esparta & 0,2 & 0,0 & 0,2 & 0,0 & 1,5 \\
\hline
\end{tabular}

Fuente: INE (2012). Los registros corresponden al año 2001.

Estos resultados también señalan un sesgo por el número de viviendas con el servicio en el estado Delta Amacuro (extremo este de la región) donde el 55,9\% lo poseen, muy por debajo al resto de las entidades; también, se aprecia similar condición en Monagas (parte central de la región) pero con un servicio que llega a cubrir el $74 \%$ del total. En general, y en consideración a estos valores, ello supone que la población restante, la que no posee acueducto o tubería, recurre al almacenamiento de agua para el consumo por los otros medios allí descritos. 
A pesar de ello, se debe mencionar que la frecuencia de abastecimiento del agua por acueducto no es regular, y puede no llegar durante días en las áreas marginales en los grandes centros poblados e, incluso, en las áreas planificadas o más organizadas de las ciudades grandes, por lo que el porcentaje de almacenaje de agua, que es una variable que no se registra, es muy alto en las viviendas, aún en los centros poblados que presentan acueductos o tuberías. Ello se convierte en el medio que asegura la presencia de las larvas que se transforman en mosquitos en los centros poblados (PAHO, 2009).

Es necesario agregar que el abastecimiento de agua tan importante para asegurar los reservorios para el consumo humano, también determina la incidencia de la enfermedad; pero se debe mencionar una amplia gama de variables que permiten la ocurrencia de la enfermedad, algunas inherentes al ámbito humano, que no son objeto de investigación en este trabajo, como la recolección de desperdicios, ya que en período lluviosos ofrecen espacios de vital importancia pare el mosquito. También influye el estado de la infraestructura y calidad del servicio de salud, aplicación de medidas preventivas para abordar al vector, su control y el tratamiento a la población infectada. Otra variable que incide en la casuística de la enfermedad, se refiere al manejo o nivel de información en que la población reconoce el problema. Por último y, finalmente, la variabilidad climática impone nuevas situaciones a la dinámica de la población, por lo que se requiere la adecuación de las medidas de ordenación del territorio y variantes que sufre el medio donde se emplazan los centros poblados.

\section{DISCUSIÓN: INTERRELACIÓN DE VARIABLES, EL DENGUE COMO UN HECHO GEOGRÁFICO}

Al cotejar la incidencia de la enfermedad en los años 2009 y 2010, período con los máximos registros de dengue en el país, y en buena medida refleja similar patrón en la región nororiental, evento que coincide en los períodos en que ocurren lluvias de registros extremos (año 2009, período muy seco; año 2010, muy lluvioso), lleva a señalar que a pesar de ser situaciones climáticas diferentes, tiene estrecha vinculación en la incidencia observada con la variable ambiental. Uno de los elementos en común que permite confirmar esta situación es el estado en que se encuentra la disponibilidad de agua, básicamente el abastecimiento de agua potable para los grupos humanos.

Durante el año 2009, los racionamientos de agua fueron extensivos en el país (Ocando, 2009), los caudales llegaron a mínimos históricos (Suite101.net, 2011). Esta sequía severa tuvo consecuencias que se evidenciaron de diversas formas, entre ellas, pérdidas agrícolas millonarias, disminución de la producción hidroeléctrica, el racionamiento eléctrico se impuso como medida de control del consumo energético interrumpiendo las actividades. Uno de los servicios que afectó a la población fue la frecuencia en el bombeo de agua potable, ello obligó a los habitantes de los centros poblados a recurrir al almacenamiento, y aún cuando las lluvias no permitían la posibilidad de crear reservorio de agua en los centros urbanos que favorecieran la presencia de larvas de los mosquitos, se multiplicaron los medios informales para resguardar el líquido para el consumo. Esta situación lleva a suponer que el almacenar agua, y en las condiciones que pudieron haberlo hecho, facilitó la presencia del mosquito y debe haber favorecido la multiplicación de la población anofelina; también esto ocurre ya que a pesar de los programas de prevención que venían ejecutándose desde el año 2008, como la nebulización de control que se hace en los espacios abiertos o en el exterior de las viviendas (Orihuela, 2008a 2008b; Red de Sociedades Científicas Médicas Venezolanas, 2011, Oletta, 2008), la casuística era alta, es decir, en el país el número de personas infectadas era elevado, y por tanto, dentro del complejo en que se desarrolla la epidemia, la movilidad de los enfermos más el número de insectos portadores del dengue deben haber sostenido e incrementado la tasa de incidencia. En la tabla 1, se puede apreciar como en Venezuela y en los estados Monagas, Anzoátegui y Nueva Esparta la incidencia era alta desde el año 2007, aún cuando los correctivos hechos en 2008, llevaron a disminuir la incidencia. Entonces cómo se explica que lloviendo menos en 2009 haya un incremento de la incidencia en la región aunque no de la misma magnitud que en el resto del país: las condiciones de humedad no estaban siendo aseguradas por las lluvias, sino a través de reservorios probablemente de tipo antrópico, aupado por el racionamiento del suministro de agua a los centros poblados. 
Durante el primer trimestre del año 2010, la situación de sequía extrema actuaba sobre el país (INAMEH), y luego se inició la temporada de lluvias en las fechas estimadas abril-mayo, (Cárdenas et al., 2003; Martelo, 2003); por otra parte, el evento Niño en el Pacifico que tuvo una fuerte intensidad en enero, va pasando de neutro a Niña (figura 3) al inicio de la temporada de lluvia, en términos comparativos, en muy poco tiempo (NOAA, 2011). En la medida que transcurrían las semanas, las frecuencias y montos de las lluvias superaron los registros históricos. Al compararlos se podía constatar que los valores de las lluvias en algunos lugares de Venezuela se estaban duplicando, como mínimo (INAMEH, 2012). Esta situación de lluvias continuas y con montos altos, se convirtió en eventos de desastres: deslaves, inundaciones, pérdidas millonarias en la producción agrícola, destrucción de redes, entre otros (El Nacional, 2011; El Universal, 2011).

Los reservorios de agua de lluvia se multiplicaron dentro y fuera de las viviendas, favoreciendo evidentemente el crecimiento de la población anofelina, y con ello la incidencia de la enfermedad. 2010 tiene la más alta incidencia de la enfermedad en Venezuela (44,7 personas/10000 hab), y los valores de la región nororiental estuvieron no por encima del promedio nacional $(68,8)$ pero sí superiores a sus series históricas (tabla 1). En esta caso, año 2010 y temporada de lluvias, aún cuando las precipitaciones eran superiores a lo normal, las fallas de abastecimiento de agua en su servicio a la población igualmente obliga a su almacenamiento y, en esta ocasión, la presencia casi continua durante semanas, de reservorios naturales en los centros poblados, sostenidos por las frecuentes precipitaciones, actuaron como medio que facilitó el incremento de la población anofelina, en una situación en que la casuística de la enfermedad era favorable para mantener la incidencia e incluso aumentarla, que fue lo ocurrido en ese año.

En 2009 y 2010 se pueden evidenciar las complejidades de la trama en la ocurrencia de la enfermedad que afecta al hombre, en este caso el dengue, y como se correlacionan los hechos: lluvias, deficiencia en el servicio de abastecimiento y número de casos para que la situación de la incidencia tome diversas formas de manifestarse.

\section{CONCLUSIÓN}

Los años 2009 y 2010, al ser extremos en la ocurrencia de las lluvias, favorecerían la presencia del mosquito que transmite la enfermedad del dengue, y los mecanismos de control del vector resultaron ineficaces al no abordar efectivamente los reservorios (que se formaron a causa de la falta de abastecimiento regular) para la reproducción de las larvas. Si bien, la incidencia no fue la más alta para la región nororiental, en las series presentadas, los registros señalan que en el año 2010 se observaron los máximos históricos de incidencia para el país.

Las lluvias ocurridas durante 2009 y 2010 se aprecian que fueron extremas, año muy seco y año muy lluvioso, y fueron el resultado de la acción de una variabilidad del clima que actúo sobre los patrones normales que producen las precipitaciones en el país.

Se puede concluir que la ocurrencia del dengue en grupos humanos, representa un hecho geográfico de mucho interés, y que aún bajo mecanismo de control, es muy compleja. La comprensión de las relaciones que se establecen entre los mosquitos y la población expuesta, pasa por considerar elementos como la variabilidad de las condiciones climáticas, el estado de los diversos servicios a la población (disponibilidad de agua potable, y otros elementos no considerados en esta investigación como la disposición de desechos, la infraestructura de salud, la educación y conciencia en educación ambiental de la población) y la reconsideración de políticas de reordenamiento del territorio con respecto al cambio climático.

Se recomienda determinar con mayor precisión la frecuencia del servicio de abastecimiento de agua, ya que aún cuando lo indicadores señalen la existencia de la forma del suministro, el almacenamiento de agua es una variable que aporta información de relevancia en los criaderos del vector.

Sería recomendable ampliar la caracterización de la ocurrencia del dengue para estos dos años para el resto de las regiones del país, y así obtener una visión espacial de la relación de la incidencia de la enfermedad. 


\section{BIBLIOGRAFÍA Y RECURSOS ELECTRÓNICOS}

CÁRDENAS, P. (2003): Impactos de los eventos El Niño-Oscilación del Sur en Venezuela. Informe Técnico. Caracas: Ministerio del Ambiente y Recursos Naturales (MARN) - Programa de las Naciones Unidas para el Desarrollo (PNUD) - Proyecto VEN/00/G31.

El NACIONAL (2011): Lluvias sobre Venezuela se pueden prolongar durante todo el mes de diciembre. Documento en línea. Disponible en: http://el-nacional.com/www/site/p contenido. php?q=nodo/169669/Ciencia\%20y\%20Tecnolog\%C3\%ADa/Lluvias-sobre-Venezuela-se-puedenprologar-durante-todo-el-mes-de-diciembre [Consultado: 2011, julio 30]

EL UNIVERSAL (2011): El Niño 2009-2010: Impactos y Consecuencias para Latinoamérica. Documento en línea. Disponible en: http://www.eluniversal.com/2010/12/06/pol ava colombia-yvenezuela_06A4822251.shtml [Consultado: 2011, julio 30]

GONZÁLEZ, S. (2010): CLIMA: 2010 entre los años más lluviosos de las últimas cuatro décadas. El Nacional. Documento en Línea. Disponible en: http://www.reportero24.com/2010/11/clima-2010-entre-losanos-mas-lluviosos-de-las-ultimas-cuatro-decadas/ [Consultado: 2011, julio 30]

HERNÁNDEZ, R. (2011): Variación porcentual de la precipitación del año 2010. Instituto Nacional de Hidrología y Meteorología. Gerencia de Meteorología. Caracas.

INSTITUTO NACIONAL DE ESTADÍSTICAS (2012): Censo Nacional de población y viviendas, año 2001. Proyecciones. Caracas.

INSTITUTO NACIONAL DE METEOROLOGÍA E HIDROlOGíA (2012): Productos y servicios. Documento en línea. Disponible en: http://www.injameh.gob.ve/index.php?pag=2010 mayo [Consultado: 2012, marzo 05]

INTERGOVERNMENTAL PANEL ON CLIMATE CHAHGE (IPCC) (2012): Working groups. Documento en línea. Disponible en: http://www.ipcc.ch/working groups/working groups.shtml [Consultado: 2011, julio 20]

MINISTERIO DE SALUD Y DESARROLLO SOCIAL (MSDS) (2006): Dengue. Tendencias Seculares. Venezuela, 1941-2003. Dirección de Vigilancia Epidemiológica. Caracas.

MINISTERIO DEL PODER POPULAR PARA LA SALUD (MPPS) (2012): Semanas epidemiológicas. Documento en Línea. Boletín Epidemiológico. Dirección General de Epidemiología. En: http://www.mpps.gob.ve Caracas: Dirección de Vigilancia Epidemiológica.

MINISTERIO DEL AMBIENTE Y DE LOS RECURSOS NATURALES RENOVABLES (MARNR) (1983): Sistemas Ambientales Naturales. Región Nororiental. Dirección General Sectorial de Ordenamiento del Territorio. Caracas.

MARTELO, M. (2003): Metodología para la selección de modelos de circulación general de la atmósfera y de escenarios climáticos a incluir en la primera comunicación nacional en cambio climático de Venezuela. Caracas: Ministerio de Ambiente y Recursos Naturales-Programa de las Naciones Unidas para el Desarrollo - Proyecto VEN/00/G31.

MINISTERIO DE SALUD Y DESARROLLO SOCIAL (MSDS) (2006): Dengue. Tendencias Seculares. Venezuela, 1941-2003. Dirección de Vigilancia Epidemiológica. Caracas.

NATIONAL OCEANIC AND ATMOSPHERIC ADMINISTRATION (NOAA) (2012): Índices de Oscilación del Sur. Documento en Línea. Disponible en: http://www.cpc.noaa.gov/data/indices/sstoi.indices [Consultado: 2011, julio 05]

ORGANIZACIÓN METEOROLÓGICA MUNDIAL (OMM) (2011): Programas científicos y técnicos de la OMM. Documento en Línea. En: http://www.wmo.int/pages/summary/progs struct es.html [Consultado: 2011, agosto 13] 
ORGANIZACIÓN PANAMERICANA DE LA SALUD (OPS) (2011): Situación de la salud en las Américas. Indicadores Básicos. Documento en Línea. Oficina Regional de la Organización de la Salud. En: http://www.paho.org/spanish/dd/ais/coredata.htm. [Consultado: 2008, agosto 02]

ORGANIZACIÓN PANAMERICANA DE LA SALUD (OPS) (2008): Reunión para Establecer una Red de Laboratorios para la Vigilancia de las Enfermedades Emergentes y Reemergentes (EER) en la Región del Cono Sur. Documento en Línea. En: http://www.paho.org/spanish/ad/dpc/cd/doc429.htm. [Consultado: 2008, agosto 27]

ORIHUELA R. (2008a): Dengue, rubéola y parotiditis en toda Venezuela entro otras repuntan. Documento en Línea. Mayo 07 de 2008. En: http://venezuelanoticia.com/archives/2811. [Consultado: 2008, agosto 26]

ORIHUELA R. (2008b): El descuido ha llevado a que se haya reproducido el dengue a nivel continental. Documento en Línea. En: http://venezuelanoticia.com/archives/32. [Consultado 2008, agosto 26]

RED DE SOCIEDADES CIENTÍFICAS MÉDICAS VENEZOLANAS (2011): Comunicado 2/2008. Documento en línea. En:http://www.rscmv.org/. [Consultado 2010, agosto 02]

OLETTA, J. (2008): Reaparecen enfermedades erradicadas hace años. Documento en Línea. Disponible en: http://venezuelanoticia.com/archives/4878 [Consultado: 2008, julio 15]

OCANDO, C. (2009): Venezuela: racionamiento del agua. Documento en línea. Disponible en: http://angelicamorabeals2.blogspot.com/2009/10/venezuelaracionamiento-del-agua.html [Consultado: 2011, agosto 21]

PANAMERIICAN HEALTH ORGANIZATION (PAHO) (2009): Guías para el diagnóstico, tratamiento, prevención y control. Documento en línea. Disponible en: http://new.paho.org/hq/dmdocuments/2011/ ndeng31570.pdf [Consultado: 2012, febrero 28]

SUITE101.net (2011): Sequía en Venezuela. Crisis energéticas, hídricas yecológicas que dejan huellas. Documento en línea. Disponible en: http://suite101.net/article/sequia-en-venezuela-a13600 [Consultado: 2011, septiembre 18] 